\title{
Acidovorax caeni sp. nov., a denitrifying species with genetically diverse isolates from activated sludge
}

\author{
Kim Heylen, Liesbeth Lebbe and Paul De Vos \\ Laboratory of Microbiology, Department of Biochemistry, Physiology and Microbiology, Ghent \\ University, K. L. Ledeganckstraat 35, B-9000 Gent, Belgium
}

Correspondence

Kim Heylen

Kim.Heylen@UGent.be
Four Gram-negative, rod-shaped, non-spore-forming, denitrifying isolates were obtained from the activated sludge of an aerobic-anaerobic wastewater treatment plant in Belgium. Analysis of repetitive sequence-based $\mathrm{PCR}$ showed that the four isolates were genetically different from each other. Results of 16S rRNA gene sequence analysis and DNA-DNA hybridization experiments indicated that these four isolates were affiliated to the genus Acidovorax and could be differentiated from all recognized species of the genus. Analysis of whole-cell proteins and results of physiological and biochemical tests allowed differentiation of the new isolates from their closest phylogenetic neighbours. These new isolates therefore represent a novel species of the genus Acidovorax, for which the name Acidovorax caeni sp. nov. is proposed. The type strain is R-24608 ${ }^{\top}$ (=LMG $24103^{\top}=$ DSM $19327^{\top}$ ).
At the time of writing, the genus Acidovorax comprises eight recognized species, which can be separated into soil and water inhabitants, Acidovorax facilis, Acidovorax delafieldii, Acidovorax temperans (Willems et al., 1990) and Acidovorax defluvii (Schulze et al., 1999), and the phytopathogenic species Acidovorax avenae and its three subspecies, Acidovorax konjaci (Willems et al., 1992), Acidovorax anthurii (Gardan et al., 2000) and Acidovorax valerianellae (Gardan et al., 2003). This separation based on occurrence and habitat is reflected in the 16S rRNA gene sequence phylogeny of these organisms, although separate phylogenetic clustering of the genus within the Comamonadaceae has been confirmed (Willems \& Gillis, 2005).

A previous cultivation-dependent study on activated sludge from an aerobic-anaerobic wastewater treatment plant used different defined growth media for specific isolation of denitrifying bacteria (Heylen et al., 2006). Nineteen denitrifiers were assigned to the genus Acidovorax based on partial 16S rRNA gene sequence analysis. The role of members of the Comamonadaceae in the removal of nitrogen in wastewater treatment plants has been recognized and described (Etchebehere et al., 2001; Gumaelius et al., 2001; Mechichi et al., 2003; Hoshino et al., 2005). Four Acidovorax-like isolates, R-24607, R-24608 ${ }^{\mathrm{T}}$, R-24613 and R-24614, were retrieved from G1M1, a mineral

The GenBank/EMBL/DDBJ accession number for the 16S rRNA gene sequence of strain $\mathrm{R}-24608^{\top}$ is $\mathrm{AM} 084006$.

A figure showing the grouping of normalized digitized SDS-PAGE patterns for members of the genus Acidovorax is available as supplementary material with the online version of this paper. medium containing $15 \mathrm{mM}$ sodium succinate, $3 \mathrm{mM}$ potassium nitrite and different vitamins. Based on partial 16S rRNA gene sequence similarity data, these new isolates could possibly represent a novel species within the environmental cluster of Acidovorax species, and were analysed further in a polyphasic study. The type strain and a second representative strain of each recognized environmental species of the genus Acidovorax, i.e. A. facilis, $A$. delafieldii, A. defluvii and A. temperans, were re-examined for phenotyping, chemotaxonomy and biochemical analysis to guarantee comparable results.

To avoid studying duplicate isolates of the same strain, genotyping by random amplified polymorphism DNA PCR analysis (Coenye et al., 2002) and repetitive sequence-based PCR analysis with REP and BOX primers (Heyrman et al., 2005) were carried out. The three fingerprint methods generated different patterns for isolates R-24607, $24608^{\mathrm{T}}$, R-24613 and R-24614, indicating genetic differences between all four isolates (data not shown). The average DNA G + C content of the four isolates, determined singly by HPLC (Mesbah et al., 1989), was $64.3 \pm 0.8 \mathrm{~mol} \%$. The nearly complete $16 \mathrm{~S}$ rRNA gene sequences of R-24607, R$24608^{\mathrm{T}}$, R-24613 and R-24614 were determined as described by Vanparys et al. (2005). Phylogenetic analysis was performed by using TREECON (Van de Peer \& De Wachter, 1994) and BioNumerics software version 4.6 after multiple alignment with CLUSTAL_X (Thompson et al., 1997). Cluster analysis according to the neighbour-joining algorithm, with or without corrections for evolutionary distances as described by Jukes \& Cantor (1969) and Kimura (1980), was in agreement with those based on the maximum-parsimony and maximum-likelihood methods. 
Strains R-24607, R-24608 ${ }^{\mathrm{T}}$, R-24613 and R-24614 clustered together with A. temperans LMG $7169^{\mathrm{T}}$, A. delafieldii LMG $5943^{\mathrm{T}}$, A. defluvii DSM $12644^{\mathrm{T}}$ and A. facilis LMG $2193^{\mathrm{T}}$, but clearly formed a separate group, supported by high bootstrap values (Fig. 1). Therefore, DNA-DNA hybridization experiments were performed within this cluster, by using a modification of the microplate method of Ezaki et al. (1989) as described by Willems et al. (2001). A hybridization temperature of $45^{\circ} \mathrm{C}$ (calculated with correction for $50 \%$ formamide) was used. First, strains R-24607, R-24608 ${ }^{\mathrm{T}}$, R-24613 and R-24614 were hybridized among themselves to substantiate the hypothesis of their relatedness at the species level. Levels of DNA-DNA relatedness among the four new isolates ranged between 78.5 and $88.5 \%( \pm 1.3-10.9 \%)$, suggesting that they represented a single species, but also confirming the genetic diversity within the strains. Strain $\mathrm{R}-24608^{\mathrm{T}}$ was further hybridized with A. temperans LMG $7169^{\mathrm{T}}$ (DNADNA relatedness of $26.7 \pm 5.6 \%$ ), A. delafieldii LMG $5943^{\mathrm{T}}$ $(23.9 \pm 5.9 \%)$, A. defluvii DSM $12644^{\mathrm{T}}(26.0 \pm 0.6 \%)$ and A. facilis LMG $2193^{\mathrm{T}}(18.2 \pm 4.8 \%)$. These results confirmed that R-24607, R-24608 ${ }^{\mathrm{T}}, \mathrm{R}-24613$ and R-24614 belong to a novel genospecies.

Cell morphology and motility were investigated by electron microscopy (Fig. 2) and phase-contrast microscopy (at a magnification of $\times 1000$ ), respectively, for cells grown on tryptone soy agar (TSA; Oxoid) for $48 \mathrm{~h}$ at $28{ }^{\circ} \mathrm{C}$. Cells were Gram stained and examined via light microscopy, and catalase and oxidase activity was determined. Utilization of carbon sources and enzyme production were tested with the API 20NE $\left(48 \mathrm{~h}, 28^{\circ} \mathrm{C}\right)$, API ZYM $\left(4 \mathrm{~h}, 28^{\circ} \mathrm{C}\right)$

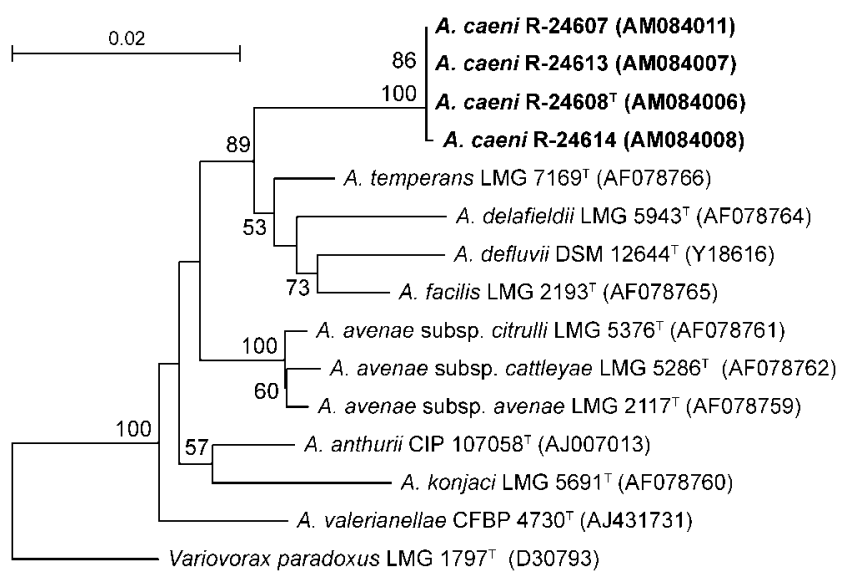

Fig. 1. Phylogenetic dendrogram obtained by neighbour-joining clustering of $16 \mathrm{~S}$ rRNA gene sequences (without correction), showing the position of strains R-24608 ${ }^{\top}, \mathrm{R}-24607, \mathrm{R}-24613$ and R-24614 among the type strains of recognized Acidovorax species. EMBL accession numbers are shown in parentheses. Variovorax paradoxus LMG $1797^{\top}$ was used as the outgroup. Relevant bootstrap values (expressed as percentages of 1000 replicates) are shown at branch points. Bar, 0.02 changes per sequence position.

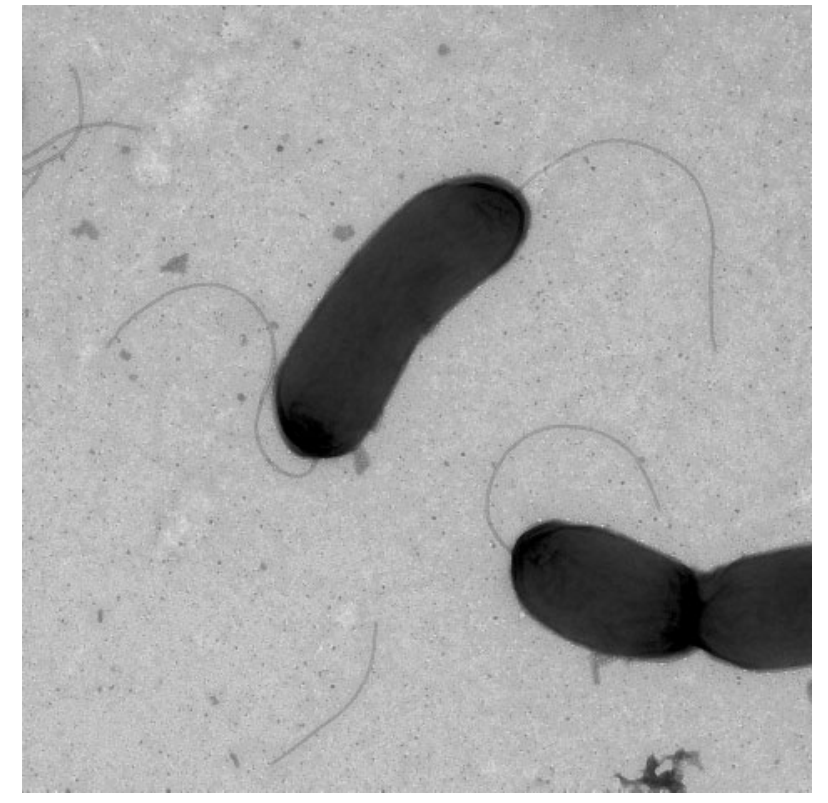

Fig. 2. Electron micrograph of cells of strain $R-24608^{\top}$, showing peritrichously flagellated rods (cell size about $0.9 \times 1.8 \mu \mathrm{m}$ ).

(bioMérieux) and Biolog $\left(24 \mathrm{~h}, 28{ }^{\circ} \mathrm{C}\right)$ systems according to the manufacturers' recommendations. The temperature range (at $4,15,28,37,45$ and $\left.52{ }^{\circ} \mathrm{C}\right), \mathrm{pH}$ range $(\mathrm{pH} 4.5-$ 10.5 at $\left.28{ }^{\circ} \mathrm{C}\right)$ and salinity range $(0.5-5 \% \mathrm{NaCl}, \mathrm{w} / \mathrm{v}$, at $28{ }^{\circ} \mathrm{C}$ ) for growth were recorded after incubation for $48 \mathrm{~h}$ in tryptone soy broth (TSB; Oxoid). The ability to denitrify was tested, as described by Smibert \& Krieg (1994), after growth for 1 week in TSB supplemented with $10 \mathrm{mM}$ potassium nitrate at $37{ }^{\circ} \mathrm{C}$ and in liquid isolation medium G1M1 at $37^{\circ} \mathrm{C}$, and was confirmed with $\mathrm{N}_{2} \mathrm{O}$ measurements, as described by Heylen et al. (2006). Lipolytic activity was determined after $72 \mathrm{~h}$ based on hydrolysis of Tween 80, as described by Sierra (1957). Differential biochemical characteristics among the test strains are given in Table 1.

After a pre-culture, all strains were incubated under identical conditions for $48 \mathrm{~h}$ at $28{ }^{\circ} \mathrm{C}$ on TSA. A loopful of well-grown cells was harvested and fatty acid methyl esters were prepared and extracted according to the standardized protocol of the Microbial Identification System (MIS; Microbial ID Inc.), and were identified by using MIDI with the TSBA database version 5.0. All strains of recognized Acidovorax species contained the characteristic fatty acids 3-hydroxyoctanoic acid $\left(\mathrm{C}_{8: 0} 3-\mathrm{OH}\right)$ and 3hydroxydecanoic acid $\left(\mathrm{C}_{10: 0} 3-\mathrm{OH}\right)$ (Willems \& Gillis, 2005). The dominant fatty acids for strains R-24607, R$24608^{\mathrm{T}}, \mathrm{R}-24613$ and R-24614 were summed feature $3(38-$ $41 \%), \mathrm{C}_{18: 1} \omega 7 c \quad(22-32 \%)$ and $\mathrm{C}_{16: 0}(25-26.5 \%)$. Unfortunately, the Sherlock MIS software could not clearly resolve summed feature 3, referring to the peaks of $\mathrm{C}_{16: 1} \omega 7 c$ and/or iso- $\mathrm{C}_{15: 0}$ 2-OH. However, Sherlock lists the closest to the observed ECL first, which was $\mathrm{C}_{16: 1} \omega 7 c$. 
Table 1. Differential physiological characteristics between strains R-24607, R-24608 ${ }^{\top}, \mathrm{R}-24613$ and R-24614 and their closest phylogenetic neighbours in the genus Acidovorax

Strains: 1, R-24607, R-24608 ${ }^{\mathrm{T}}$, R-24613 and R-24614; 2, A. defluvii DSM $12644^{\mathrm{T}}$ and DSM $12578 ; 3$, A. delafieldii LMG $5943^{\mathrm{T}}$ and LMG 8909; 4, A. facilis LMG $2193^{\mathrm{T}}$ and LMG 6598; 5, A. temperans LMG $7169^{\mathrm{T}}$ and LMG 7163. Data are from this study unless otherwise indicated. +, Positive; $\mathrm{w}$, weakly positive; - , negative; $\mathrm{v}$, variable (test result for the type strain given in parentheses). All were positive for oxidase, leucine arylamidase and assimilation of methyl pyruvate, monomethyl succinate, $\beta$-hydroxybutyric acid, $\alpha$-ketovaleric acid and DL-lactic acid. All were negative for arginine dihydrolase, urease, lipase (C14), valine arylamidase, cystine arylamidase, $\alpha$-chymotrypsin, acid phosphatase, $\alpha$-galactosidase, $\beta$-galactosidase, $\beta$-glucuronidase, $\alpha$-glucosidase, $\beta$-glucosidase (aesculin hydrolysis), $N$-acetyl- $\beta$-glucosamidase, $\alpha$-mannosidase, $\alpha$-fucosidase, indole production, glucose fermentation and assimilation of L-mannose, phenylacetic acid, $\mathrm{N}$ acetyl-D-galactosamine, adonitol, cellobiose, L-fucose, gentiobiose, myo-inositol, $\alpha$-D-lactose, lactulose, D-melibiose, methyl $\beta$-glucoside, D-raffinose, sucrose, trehalose, turanose, xylitol, citric acid, Dgalactonic acid lactone, D-galacturonic acid, D-glucosaminic acid, Dglucuronic acid, saccharic acid, glucuronamide, L-histidine, inosine, uridine, thymidine, phenyl ethylamine, putrescine, 2-aminoethanol, 2,3-butanediol, DL- $\alpha$-glycerol phosphate and glucose 1-phosphate.

\begin{tabular}{|c|c|c|c|c|c|}
\hline Characteristic & 1 & 2 & 3 & 4 & 5 \\
\hline Catalase & + & $\mathrm{v}(+)$ &,$- v^{*}$ & + & + \\
\hline Hydrolysis of Tween 40 & + & $\mathrm{V}(-)$ & + & $\mathrm{v}(-)$ & + \\
\hline Hydrolysis of Tween 80 & + & $+\dagger$ & + & $\mathrm{v}(-)$ & + \\
\hline Nitrate reduction & + & $\mathrm{v} \ddagger$ & + & + & + \\
\hline Denitrification & + & $\mathrm{V} \ddagger$ & - & - & + \\
\hline Alkaline phosphatase & $\mathrm{w}$ & - & + & $\mathrm{W}$ & + \\
\hline Esterase $(\mathrm{C} 4)$ & $\mathrm{W}$ & + & - & + & $\mathrm{w}$ \\
\hline Esterase lipase (C8) & + & $\mathrm{w}$ & + & + & + \\
\hline Trypsin & + & - & - & - & - \\
\hline $\begin{array}{l}\text { Naphthol-AS-BI- } \\
\text { phosphohydrolase }\end{array}$ & + & - & - & - & - \\
\hline Indole production & + & - & - & - & - \\
\hline Gelatinase & - & - &,$- \mathrm{V}^{*} \mathrm{~V}$ & $(-),+^{*}$ & - \\
\hline \multicolumn{6}{|l|}{ Assimilation of: } \\
\hline L-Arabinose & - & - & + &,$-+^{\star}$ & - \\
\hline D-Mannose & - & - & + &,$-+^{\star}$ & - \\
\hline D-Mannitol & - & - & + &,$-+^{\star}$ & + \\
\hline$N$-Acetylglucosamine & - & - & - & + & - \\
\hline Maltose & - & - & - & + & - \\
\hline Potassium gluconate & - & - & + &,$-+^{*}$ & - \\
\hline Capric acid & - & - &,$- \mathrm{v}^{*}$ & - &,$- \mathrm{v}^{*}$ \\
\hline Adipic acid & - & + & - & - & - \\
\hline Malate & + & - & $\mathrm{V}$ & - & + \\
\hline Trisodium citrate & - & - &,$- \mathrm{v}^{*}$ & - & - \\
\hline$\alpha$-Cyclodextrin & $\mathrm{v}(-)$ & - & $\mathrm{V}(-)$ & - & - \\
\hline Dextrin & - & - & $\mathrm{v}(-)$ & - & - \\
\hline Glycogen & + & - & - & - &,$+-^{\star}$ \\
\hline D-Arabitol & - & - & + & - & $\mathrm{V}(-)$ \\
\hline i-Erythritol & $\mathrm{V}(-)$ & - & - & - & - \\
\hline D-Fructose & - & - & + & $\mathrm{v}(-)$ & + \\
\hline D-Galactose & - & $\mathrm{v}(+)$ & + & - & - \\
\hline
\end{tabular}

\begin{tabular}{|c|c|c|c|c|c|}
\hline Characteristic & 1 & 2 & 3 & 4 & 5 \\
\hline$\alpha$-D-Glucose & - & - & $\mathrm{v}(\mathrm{v}) \|$ &,$-+^{\star}$ & + \\
\hline D-Psicose & - & - & $\mathrm{V}(-)$ & $\mathrm{V}(-)$ & + \\
\hline D-Sorbitol & - & - & + & - & + \\
\hline Acetic acid & $\mathrm{V}(+)$ & - & - & - & - \\
\hline cis-Aconitic acid & $\mathrm{V}(+)$ & - & $\mathrm{v}(+)$ & - & - \\
\hline Formic acid & + & - & $\mathrm{v}(+)$ & - & - \\
\hline D-Gluconic acid & - & - & + & - & - \\
\hline$\alpha$-Hydroxybutyric acid & + & $\mathrm{V}(-)$ & + & - & + \\
\hline$\gamma$-Hydroxybutyric acid & + & $\mathrm{V}(-)$ & + & - & + \\
\hline $\begin{array}{l}p \text {-Hydroxyphenylacetic } \\
\text { acid }\end{array}$ & - & - & + & - & - \\
\hline Itaconic acid & - & - & - & - & - \\
\hline$\alpha$-Ketobutyric acid & + & $\mathrm{V}(-)$ & + & $\mathrm{V}(-)$ & + \\
\hline$\alpha$-Ketoglutaric acid & + & $\mathrm{V}(+)$ & + & $\mathrm{V}(-)$ & + \\
\hline Malonic acid & $\mathrm{V}(-)$ & - & - & $\mathrm{v}(-)$ & - \\
\hline Propionic acid & + & - & $\mathrm{V}(+)$ & - & $\mathrm{v}(-)$ \\
\hline Quinic acid & - & - & + & - & - \\
\hline Sebacic acid & $\mathrm{v}(+)$ & + & + & $\mathrm{V}(-)$ & + \\
\hline Succinic acid & + & + & + & $\mathrm{v}(-)$ & + \\
\hline Bromosuccinic acid & + & - & $\mathrm{v}(+)$ & $\mathrm{v}(-)$ & + \\
\hline Succinamic acid & $\mathrm{V}(-)$ & - & $\mathrm{v}(+)$ & $\mathrm{v}(-)$ & + \\
\hline Alaninamide & $\mathrm{V}(-)$ & $\mathrm{v}(+)$ & + & $\mathrm{V}(-)$ & + \\
\hline D-Alanine & + & - & + & $\mathrm{v}(-)$ & + \\
\hline L-Alanine & + & + & + & $\mathrm{V}(-)$ & + \\
\hline L-Alanyl glycine & + & $\mathrm{v}(+)$ & + & + & + \\
\hline L-Asparagine & $\mathrm{V}(-)$ & - & + & - & + \\
\hline L-Aspartic acid & + & - & + & - & + \\
\hline L-Glutamic acid & + & - & + & - & + \\
\hline Glycyl L-aspartic acid & - & - & + & $\mathrm{V}(-)$ & - \\
\hline Glycyl L-glutamic acid & - & - & + & - & $\mathrm{v}(+)$ \\
\hline Hydroxy L-proline & - & - & + & - & - \\
\hline L-Leucine & + & - & + & $\mathrm{V}(-)$ & + \\
\hline L-Ornithine & - & - & $\mathrm{v}(+)$ & - & $\mathrm{v}(-)$ \\
\hline L-Phenylalanine & - & - & $\mathrm{v}(+)$ & + & + \\
\hline L-Proline & + & - & + & + & + \\
\hline L-Pyroglutamic acid & + & - & $\mathrm{v}(+)$ & + & + \\
\hline D-Serine & $\mathrm{V}(-)$ & - & - & - & - \\
\hline L-Serine & + & - & + &,$-+^{\star}$ & - \\
\hline L-Threonine & + & $\mathrm{V}(-)$ & + & - & $\mathrm{v}(-)$ \\
\hline DL-Carnitine & - & - & $\mathrm{v}(+)$ & $\mathrm{v}(+)$ & - \\
\hline$\gamma$-Aminobutyric acid & - & - & + & $\mathrm{v}(+)$ & $\mathrm{v}(-)$ \\
\hline Urocanic acid & - & - & + & - & - \\
\hline Glycerol & + & $\mathrm{v}(+)$ & + & - & + \\
\hline Glucose 6-phosphate & $\mathrm{V}(-)$ & - & $\mathrm{v}(+)$ & - & - \\
\hline
\end{tabular}

${ }^{\star}$ Data from Willems \& Gillis (2005).

$\dagger$ DSM $12644^{\mathrm{T}}$ scored negative for Tween 80 hydrolysis in the Biolog screening (after $24 \mathrm{~h}$ ), but positive when grown on Sierra medium for $72 \mathrm{~h}$.

¥In contrast to DSM 12578 , DSM $12644^{\mathrm{T}}$ does not reduce nitrate to nitrite or further (denitrification) under the tested growth conditions (in G1M1 and in supplemented TSB). With the API 20NE gallery, the two strains tested positive for nitrate reduction and denitrification. §Data from Schulze et al. (1999).

IIA. delafieldii LMG $5943^{\mathrm{T}}$ tested positive for glucose assimilation with API 20NE but negative with Biolog. 
In addition, comparison of the fatty acid data in the literature for the type strains of recognized species of the genus Acidovorax (Willems et al., 1990; Schulze et al., 1999) and our data on the same strains suggests $C_{16: 1} \omega 7 c$ as the major fatty acid for this peak. No characteristic fatty acids for the novel genospecies were detected (Table 2).

SDS-PAGE analysis of whole-cell proteins was performed on aerobically grown cells after incubation at $28{ }^{\circ} \mathrm{C}$ for $40 \mathrm{~h}$ on phosphate-buffered nutrient agar ( $\mathrm{pH} \mathrm{6.8).} \mathrm{An}$ SDS-PAGE banding pattern for all strains was generated according to a standardized protocol (Pot et al., 1994). Pearson's correlation similarity coefficients were clustered with UPGMA and analysed with the co-phenetic correlation method in BioNumerics version 4.6 (see Supplementary Fig. S1 in IJSEM Online). The different strains of each species grouped together, supported with high co-phenetic correlation values. Although strains R24607, R-24608 ${ }^{\mathrm{T}}$, R-24613 and R-24614 demonstrated significant variation in whole-cell protein profiles, they formed a distinct group, separate from recognized species of the genus Acidovorax.
Based on the polyphasic data presented here, strains R$24607, \mathrm{R}-24608^{\mathrm{T}}, \mathrm{R}-24613$ and $\mathrm{R}-24614$ are considered to represent a novel species of the genus Acidovorax, for which the name Acidovorax caeni sp. nov. is proposed.

\section{Description of Acidovorax caeni sp. nov.}

Acidovorax caeni (cae'ni. L. gen. neut. n. caeni of sludge).

After $48 \mathrm{~h}$, colonies are round, smooth and yellow-brown. Cells are motile, non-spore-forming rods $(0.9 \times 1.8 \mu \mathrm{m})$. Gram-negative and catalase- and oxidase-positive. Growth is observed at $15-37{ }^{\circ} \mathrm{C}$ but not at 4 or $45-52{ }^{\circ} \mathrm{C}$, at $\mathrm{pH}$ 5.5-10.5, but not at $\mathrm{pH} 4.5-5$, and at salt concentrations of $0.5-2 \% \mathrm{NaCl}(\mathrm{w} / \mathrm{v})$, but not at $3-5 \% \mathrm{NaCl}$. Anaerobic respiration and growth are possible through denitrification. Positive for the following enzyme activities: hydrolysis of Tweens 40 and 80 , esterase lipase, trypsin, naphthol-AS-BI-phosphohydrolase and production of indole. Malate, glycogen, formic acid, $\alpha$-hydroxybutyric acid, $\beta$-hydroxybutyric acid, $\alpha$-ketobutyric acid, $\alpha$-ketoglutaric acid, propionic acid, succinic acid, bromosuccinic

Table 2. Fatty acid content of strains $\mathrm{R}-24607, \mathrm{R}-24608^{\top}, \mathrm{R}-24613$ and $\mathrm{R}-24614$ and their closest phylogenetic neighbours in the genus Acidovorax

Strains: 1, R-24607, R-24608 ${ }^{\mathrm{T}}$, R-24613 and R-24614; 2, A. defluvii DSM $12644^{\mathrm{T}}$ and DSM 12578; 3, A. delafieldii LMG 5943 ${ }^{\mathrm{T}}$ and LMG 8909; 4 , A. facilis LMG $2193^{\mathrm{T}}$ and LMG 6598; 5, A. temperans LMG $7169^{\mathrm{T}}$ and LMG 7163. All data were obtained in the present study. Values are percentages of the total fatty acid content. -, Not present; tr, trace component $(<1 \%)$.

\begin{tabular}{|c|c|c|c|c|c|}
\hline Fatty acid & 1 & 2 & 3 & 4 & 5 \\
\hline $\mathrm{C}_{8: 0} 3-\mathrm{OH}$ & $0.78-1.19$ & $\operatorname{tr}$ & $\operatorname{tr}$ & $0.88-1.87$ & $1.04-1.27$ \\
\hline $\mathrm{C}_{9: 0} 3-\mathrm{OH}$ & - & $0.32-1.14$ & - & - & $\operatorname{tr}$ \\
\hline $\mathrm{C}_{11: 0}$ & - & $\operatorname{tr}$ & - & - & $\operatorname{tr}$ \\
\hline $\mathrm{C}_{10: 0} 3-\mathrm{OH}$ & $2.81-4.32$ & $3.13-3.90$ & $3.16-3.59$ & $2.78-6.78$ & $3.96-4.25$ \\
\hline Unknown 11.799 & - & - & - & $\operatorname{tr}$ & - \\
\hline $\mathrm{C}_{12: 0}$ & $2.64-3.24$ & $2.18-2.39$ & $2.77-2.89$ & $2.91-5.48$ & $5.52-5.72$ \\
\hline iso- $\mathrm{C}_{11: 0} 3-\mathrm{OH}$ & - & $\operatorname{tr}$ & - & - & - \\
\hline $\mathrm{C}_{11: 0} 3-\mathrm{OH}$ & - & $\operatorname{tr}$ & - & - & - \\
\hline $\mathrm{C}_{13: 0}$ & - & $\operatorname{tr}$ & - & - & $1.08-3.46$ \\
\hline $\mathrm{C}_{14: 0}$ & $\operatorname{tr}$ & $1.57-1.63$ & $3.25-3.37$ & $3.09-.374$ & $0.99-3.53$ \\
\hline $\mathrm{C}_{15: 1} \omega 6 c$ & - & $1.15-11.97$ & - & - & $3.23-4.51$ \\
\hline iso- $\mathrm{C}_{15: 0}$ & - & $\operatorname{tr}$ & - & - & - \\
\hline Summed feature $3^{*}$ & $38.24-41.16$ & $40.57-48.05$ & $39.35-41.32$ & $43.68-43.71$ & $46.92-47.15$ \\
\hline $\mathrm{C}_{16: 0}$ & $24.90-26.41$ & $17.70-22.78$ & $26.33-27.99$ & $25.51-29.24$ & $19.35-20.99$ \\
\hline iso- $\mathrm{C}_{17: 1} \omega 9 c$ & - & $\operatorname{tr}$ & - & - & - \\
\hline iso- $\mathrm{C}_{17: 0}$ & $\operatorname{tr}$ & $\operatorname{tr}$ & - & - & - \\
\hline $\mathrm{C}_{17: 0}$ cyclo & - & - & $\operatorname{tr}$ & $\operatorname{tr}$ & - \\
\hline $\mathrm{C}_{17: 1} \omega 8 c$ & - & tr & - & - & - \\
\hline $\mathrm{C}_{17: 1} \omega 6 c$ & - & $1.41-2.09$ & - & - & $\operatorname{tr}$ \\
\hline $\mathrm{C}_{17: 0}$ & $\operatorname{tr}$ & $4.87-6.27$ & $\operatorname{tr}$ & $\operatorname{tr}$ & $2.10-3.7$ \\
\hline $\mathrm{C}_{18: 1} \omega 7 c$ & $22.24-31.96$ & $8.07-11.04$ & $19.42-22.73$ & $11.78-16.56$ & $9.12-9.64$ \\
\hline $\mathrm{C}_{18: 0}$ & $\operatorname{tr}$ & - & $\operatorname{tr}$ & $\operatorname{tr}$ & - \\
\hline Methyl $\mathrm{C}_{18: 1} \omega 7 c$ & $\operatorname{tr}$ & $\operatorname{tr}$ & $\operatorname{tr}$ & - & - \\
\hline Summed feature $7 \dagger$ & $\operatorname{tr}$ & - & - & - & - \\
\hline
\end{tabular}

*Summed feature 3 comprises $\mathrm{C}_{16: 1} \omega 7 c$ and/or iso- $\mathrm{C}_{15: 0} 2-\mathrm{OH}$.

$\uparrow$ Summed feature 7 comprises $\mathrm{C}_{19: 1} \omega 6 c$ and/or $\mathrm{C}_{19: 0}$ cyclo. 
acid, D-alanine, L-alanine, L-alanyl glycine, L-aspartic acid, L-glutamic acid, L-leucine, L-proline, L-pyroglutamic acid, L-serine, L-threonine, methyl pyruvate, monomethyl succinate, $\beta$-hydroxybutyric acid, $\alpha$-ketovaleric acid, DL-lactic acid and glycerol can be used as carbon sources. Can be differentiated from the type strains of its closest phylogenetic neighbours, A. defluvii, A. delafieldii, A. facilis and A. temperans, through SDS-PAGE analysis of whole-cell proteins, by the ability to produce indole and by the presence of trypsin and naphthol-AS-BI-phosphohydrolase.

The type strain, R-24608 ${ }^{\mathrm{T}}\left(=\mathrm{LMG} 24103^{\mathrm{T}}=\mathrm{DSM} 19327^{\mathrm{T}}\right)$, has a DNA G $+C$ content of $65.7 \mathrm{~mol} \%$ and was isolated from activated sludge from an aerobic-anaerobic wastewater treatment plant (Bourgoyen-Ossemeersen) in Gent, Belgium. Due to the genetic variation within this species, R-24607, R-24613 and R-24614 were also deposited in the BCCM/LMG collection with strain numbers LMG 24104, LMG 24105 and LMG 24106, respectively.

\section{Acknowledgements}

This work was supported by project G.O.A. 1205073 (2003-2008) of the Ministerie van de Vlaamse Gemeenschap, Bestuur Wetenschappelijk Onderzoek (Belgium) and the FWO project G20156.02.

\section{References}

Coenye, T., Spilker, T., Martin, A. \& LiPuma, J. J. (2002). Comparative assessment of genotyping methods for epidemiologic study of Burkholderia cepacia genomovar III. J Clin Microbiol 40, 3300-3307.

Etchebehere, C., Errazquin, I., Barrandeguy, E., Dabert, P., Moletta, R. \& Muxi, L. (2001). Evaluation of the denitrifying microbiota of anoxic reactors. FEMS Microbiol Ecol 35, 259-265.

Ezaki, T., Hashimoto, Y. \& Yabuuchi, E. (1989). Fluorometric deoxyribonucleic acid-deoxyribonucleic acid hybridization in microdilution wells as an alternative to membrane filter hybridization in which radioisotopes are used to determine genetic relatedness among bacterial strains. Int J Syst Bacteriol 39, 224-229.

Gardan, L., Dauga, C., Prior, P., Gillis, M. \& Saddler, G. S. (2000). Acidovorax anthurii sp. nov., a new phytopathogenic bacterium which causes bacterial leaf-spot of anthurium. Int J Syst Evol Microbiol 50, 235-246.

Gardan, L., Stead, D. E., Dauga, C. \& Gillis, M. (2003). Acidovorax valerianellae sp. nov., a novel pathogen of lamb's lettuce [Valerianella locusta (L.) Laterr.]. Int J Syst Evol Microbiol 53, 795-800.

Gumaelius, L., Magnusson, G., Pettersson, B. \& Dalhammar, G. (2001). Comamonas denitrificans sp. nov., an efficient denitrifying bacterium from activated sludge. Int J Syst Evol Microbiol 51, 999-1006.

Heylen, K., Vanparys, B., Wittebolle, L., Verstraete, W., Boon, N. \& De Vos, P. (2006). Cultivation of denitrifying bacteria: optimization of isolation conditions and diversity study. Appl Environ Microbiol 72, 2637-2643.

Heyrman, J., Verbeeren, J., Schumann, P., Swings, J. \& De Vos, P. (2005). Six novel Arthrobacter species isolated from deteriorated mural paintings. Int J Syst Evol Microbiol 55, 1457-1464.

Hoshino, T., Terahara, T., Tsuneda, S., Hirata, A. \& Inamori, Y. (2005). Molecular analysis of microbial population transition associated with the start of denitrification in a wastewater treatment process. J Appl Microbiol 99, 1165-1175.
Jukes, T. H. \& Cantor, C. R. (1969). Evolution of protein molecules. In Mammalian Protein Metabolism, pp. 21-132. Edited by H. N. Munro. New York: Academic Press.

Kimura, M. (1980). A simple method for estimating evolutionary rates of base substitutions through comparative studies of nucleotide sequences. J Mol Evol 16, 111-120.

Mechichi, T., Stackebrandt, E. \& Fuchs, G. (2003). Alicycliphilus denitrificans gen. nov., sp. nov., a cyclohexanol-degrading, nitratereducing $\beta$-proteobacterium. Int J Syst Evol Microbiol 53, 147-152.

Mesbah, M., Premachandran, U. \& Whitman, W. B. (1989). Precise measurement of the $\mathrm{G}+\mathrm{C}$ content of deoxyribonucleic acid by high-performance liquid chromatography. Int J Syst Bacteriol 39, 159-167.

Pot, B., Vandamme, P. \& Kersters, K. (1994). Analysis of electrophoretic whole organism protein fingerprints. In Chemical Methods in Prokaryotic Systematics, pp. 493-521. Edited by M. Goodfellow \& A. G. O’Donnell. Chichester: Wiley.

Schulze, R., Spring, S., Amann, R., Huber, I., Ludwig, W., Schleifer, K.-H. \& Kämpfer, P. (1999). Genotypic diversity of Acidovorax strains isolated from activated sludge and description of Acidovorax defluvii sp. nov. Syst Appl Microbiol 22, 205-214.

Sierra, G. (1957). A simple method for the detection of lipolytic activity of micro-organisms and some observations on the influence of the contact between cells and fatty substrates. Antonie van Leeuwenhoek 23, 15-22.

Smibert, R. M. \& Krieg, N. R. (1994). General characterization. In Manual of Methods for General Microbiology, pp. 409-443. Edited by P. Gerhardt, R. G. E. Murray, R. N. Costilow, E. W. Nester, W. A. Wood, N. R. Krieg \& G. B. Phillips. Washington, DC: American Society for Microbiology.

Thompson, J. D., Gibson, T. J., Plewniak, F., Jeanmougin, F. \& Higgins, D. G. (1997). The CLUSTAL_X windows interface: flexible strategies for multiple sequence alignment aided by quality analysis tools. Nucleic Acids Res 25, 4876-4882.

Van de Peer, Y. \& De Wachter, R. (1994). TREECON for Windows: a software package for the construction and drawing of evolutionary trees for the Microsoft Windows environment. Comput Appl Biosci 10, 569-570.

Vanparys, B., Heylen, K., Lebbe, L. \& De Vos, P. (2005). Pedobacter caeni sp. nov., a novel species isolated from a nitrifying inoculum. Int J Syst Evol Microbiol 55, 1315-1318.

Willems, A. \& Gillis, M. (2005). Genus II. Acidovorax Willems, Falsen, Pot, Jantzen, Hoste, Vandamme, Gillis, Kersters and De Ley 1990, $394^{\mathrm{VP}}$. In Bergey's Manual of Systematic Bacteriology, 2nd edn, vol. 2, part C, pp. 696-703. Edited by D. J. Brenner, N. R. Krieg, J. T. Staley \& G. M. Garrity. New York: Springer.

Willems, A., Falsen, E., Pot, B., Jantzen, E., Hoste, B., Vandamme, P., Gillis, M., Kersters, K. \& De Ley, J. (1990). Acidovorax, a new genus for Pseudomonas facilis, Pseudomonas delafieldii, E. Falsen (EF) group 13, EF group 16, and several clinical isolates, with the species Acidovorax facilis comb. nov., Acidovorax delafieldii comb. nov., and Acidovorax temperans sp. nov. Int J Syst Bacteriol 40, 384-398.

Willems, A., Goor, M., Thielemans, S., Gillis, M., Kersters, K. \& De Ley, J. (1992). Transfer of several phytopathogenic Pseudomonas species to Acidovorax avenae subsp. avenae subsp. nov., comb. nov., Acidovorax avenae subsp. citrulli, Acidovorax avenae subsp. cattleyae, and Acidovorax konjaci. Int J Syst Bacteriol 42, 107-119.

Willems, A., Doignon-Bourcier, F., Goris, J., Coopman, R., de Lajudie, P., De Vos, P. \& Gillis, M. (2001). DNA-DNA hybridization study of Bradyrhizobium strains. Int J Syst Evol Microbiol 51, 1315-1322. 